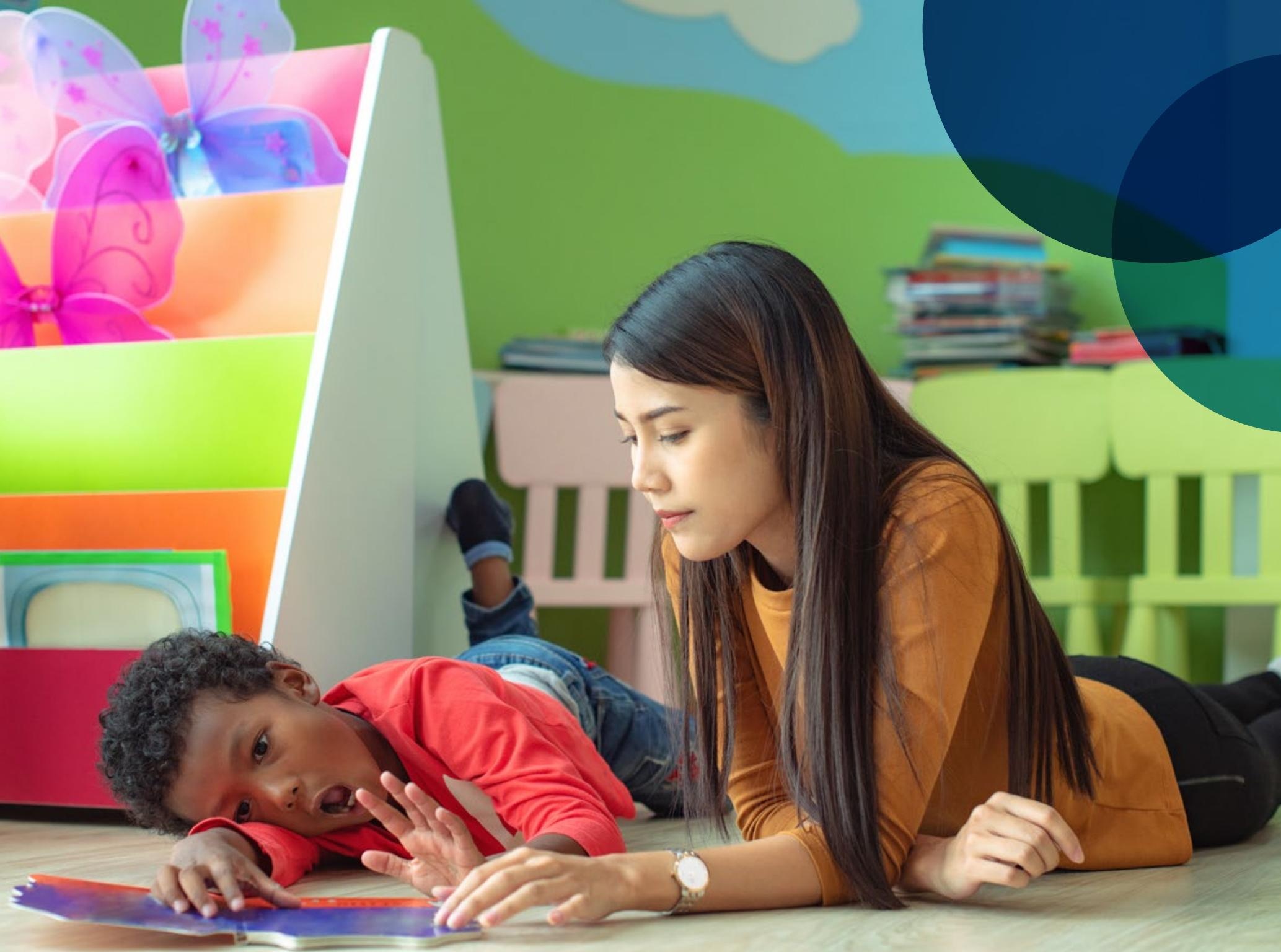

\title{
New developmentally appropriate diagnostic criteria need to be established to identify ADHD early in preschoolers
}

\section{By Jessica K. Edwards}

Attention-deficit/hyperactivity disorder (ADHD) typically emerges during preschool years and in a subset of children, can persist into adolescence ${ }^{1}$. Early identification might help promote a favourable ADHD trajectory ${ }^{2}$, but the current predictors of ADHD persistence are insufficient. In 2019, Jeffrey Halperin and David Marks compiled a Practitioner Review for the Journal of Child Psychology and Psychiatry on ADHD in preschool children. Their review provides up-to-date information on how to evaluate and treat ADHD in preschoolers and an assessment of the latest findings from randomized controlled trials of nonpharmacological and pharmacological interventions.
Stand out findings include; that while hyperactiveimpulsive symptoms extend down to age 3 years, inattention symptoms that are defined by the $D S M-V_{1}$, less accurately differentiate between preschoolers with and without ADHD. In addition, data suggest that preschoolers who do not meet the full diagnostic criteria for ADHD are more likely to develop the disorder or experience other ongoing difficulties than outgrow it ${ }^{3,4}$ : Halperin and Marks recommend that clinical attention is given to this group of children with sub-threshold ADHD, to intervene early where necessary, and to encourage a healthy trajectory. 
In terms of treatment recommendations, the researchers consider that Behavioural Parent Training should be the first-line treatment for ADHD in preschoolers. Although they acknowledge that medication can reduce symptom severity, the efficacy and safety profiles of medication for ADHD are less desirable in preschoolers compared to those of school age $\mathrm{e}^{5}$. They thus propose that this treatment modality is reserved for those who do not show sufficient improvement after behavioural intervention.

Going forward, Halperin and Marks hope to see the establishment of appropriate diagnostic criteria for preschoolers with ADHD and the identification of moderators of treatment responses. Most importantly, they explain that new interventions are needed that have long-lasting benefits, such that they can alter the long-term trajectory of ADHD in children.

\section{Referring to:}

Halperin, J.M. \& Marks, D.J. (2019), Practitioner Review: Assessment and treatment of preschool children with attention-deficit/hyperactivity disorder. J. Child Psychol. Psychiatr. 60: 930-943. doi: 10.111/jcpp.13014.

\section{See also:}

Center for Medicare \& Medicaid Services (2015). Stimulant use and related medications: Use in pediatric patients. Available from: https://www. cms.gov/Medicare-Medicaid-Coordination/ Fraud-Prevention/Medicaid-Integrity-Education/ Pharmacy-Education-Materials/Downloads/stimpediatric-factsheetı1-14.pdf.

\section{Glossary:}

Behavioural Parent Training: An integrated program typically involving family systems theory, social learning principles, and operant conditioning to address behavioural issues in pre-school and school-age children. As described by Halperin and Marks, most programs incorporate positive reinforcement, ignoring of low-level, benign provocation, and application of clear, consistent, and constructive consequences in response to undesirable behaviour.

\section{References:}

${ }^{1}$ American Psychiatric Association (2013), Diagnostic and statistical manual of mental disorders, 5th ed. Washington, DC: American Psychiatric Association.

${ }^{2}$ Sonuga-Barke, E.J. et al. (2010), Developmental phenotypes and causal pathways in attentiondeficit/hyperactivity disorder: Potential targets for early intervention? J. Child Psychol. Psychiatr. 51: 368-389. doi: 10.1111/j.1469-7610.2009.02195.x.

3Law, E.C. et al. (2014), Attention-deficit/ hyperactivity disorder in young children: Predictors of diagnostic stability. Pediatrics, 133, 659-667. doi: 10.1542/peds.2013-3433.

${ }^{4}$ Lahey, B.B. et al. (2016), Predictors of adolescent outcomes among 4-6-year-old children with attention-deficit/hyperactivity disorder. J. Abnorm. Psychol. 125: 168-181. doi: 10.1037/abnooooo86

${ }^{5}$ Riddle, M.A. et al. 2013. The preschool attentiondeficit/hyperactivity disorder treatment study (PATS) 6-year follow-up. J. Am. Acad. Child Adolesc. Psychiatry. 5 2: 264-278. doi: 10.1016/j. jaac.2012.12.007.

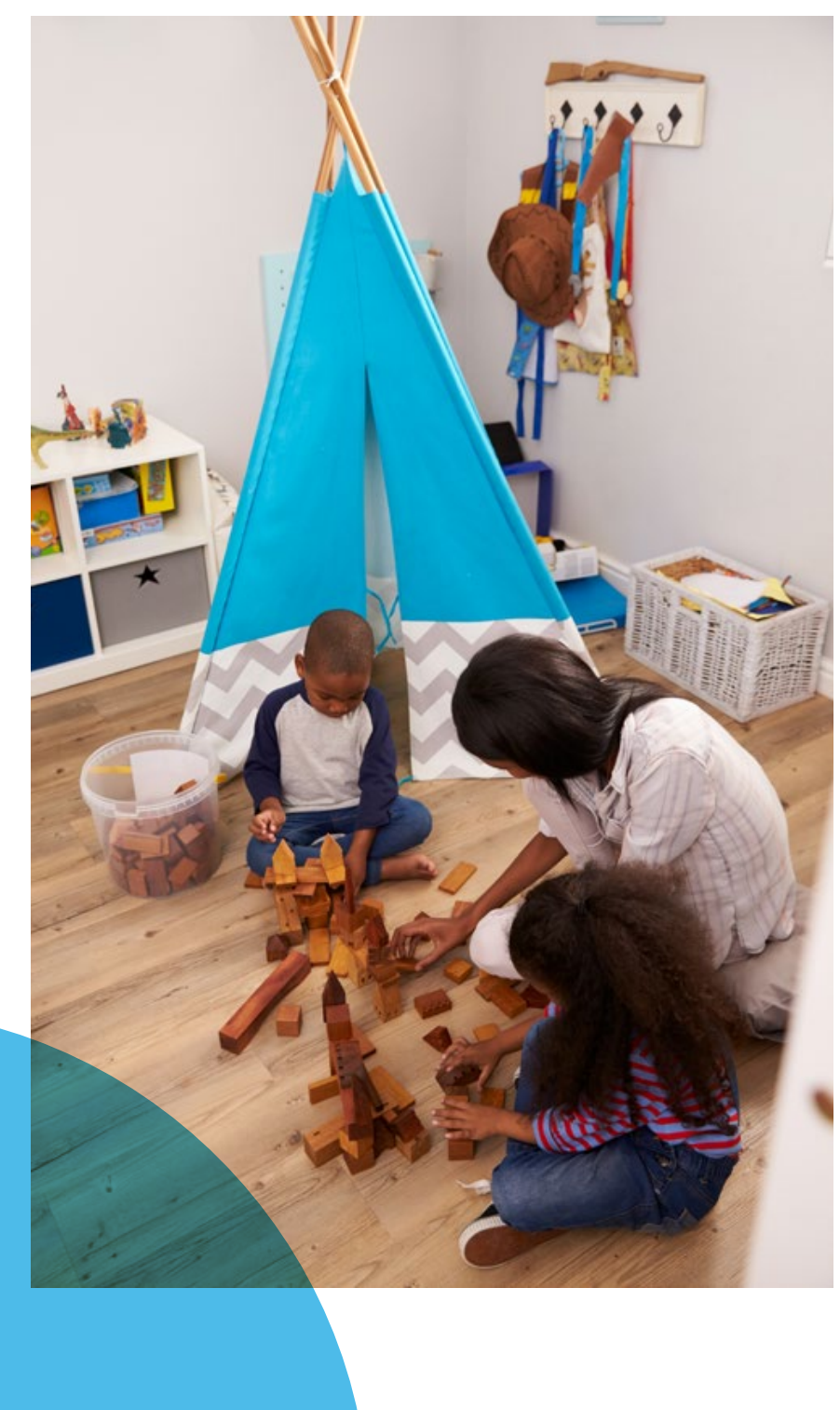

